\title{
RursuSpicae
}

Transmission, réception et réécriture de textes, de I'Antiquité au Moyen Âge

$2 \mid 2019$

Le Physiologus. Manuscrits anciens et tradition médiévale

\section{Une transposition exemplaire. À propos du rapport entre texte et illustration dans le Physiologus de Bruxelles (Ms. KBR 10066-77. Meuse, fin du X $\mathrm{X}^{\mathrm{e}}$ s. ?)}

An Exemplary Transposition: The Relationship between Text and Image in The Brussels Physiologus (MS KBR 1066-77; Meuse, end of the tenth century?)

Jacqueline Leclercq-Marx

\section{(2) OpenEdition}

Journals

\section{Édition électronique}

URL : http://journals.openedition.org/rursuspicae/978

DOI : $10.4000 /$ rursuspicae. 978

ISSN : 2557-8839

Éditeur :

Université Nice-Sophia Antipolis, IRHT - Institut de recherche et d'histoire des textes

\section{Référence électronique}

Jacqueline Leclercq-Marx, « Une transposition exemplaire. À propos du rapport entre texte et illustration dans le Physiologus de Bruxelles (Ms. KBR 10066-77. Meuse, fin du Xe s. ?) 》, RursuSpicae [En ligne], 2 | 2019, mis en ligne le 20 décembre 2019, consulté le 29 mars 2021. URL : http:// journals.openedition.org/rursuspicae/978; DOI : https://doi.org/10.4000/rursuspicae.978 


\section{Une transposition exemplaire. À propos du rapport entre texte et illustration dans le Physiologus de Bruxelles (Ms. KBR 10066-77. Meuse, fin $\mathrm{du} \mathrm{X}^{\mathrm{e}} \mathrm{s.}$ ?)}

An Exemplary Transposition: The Relationship between Text and Image in The Brussels Physiologus (MS KBR 1066-77; Meuse, end of the tenth century?)

Jacqueline Leclercq-Marx

\section{Introduction}

1 Le Physiologus dit de Bruxelles appartient au noyau le plus ancien (f. 80-162) d'un recueil de textes médiévaux de natures et d'époques différentes. Comme les autres avec lesquels on peut le regrouper ${ }^{1}$, il se situe dans une tranche chronologique située entre le $\mathrm{X}^{\mathrm{e}}$ et le $\mathrm{XI}^{\mathrm{e}}$ siècles, et doit vraisemblablement être daté de la fin du $\mathrm{X}^{\mathrm{e}}$ siècle. Par ailleurs, le style de ses illustrations l'apparente étroitement à la Psychomachie de Prudence qui le précède dans le même cahier². La Psychomachie et le Physiologus sont toutefois d'inégale ampleur : sur 47 feuillets cotés de 112 à 156 (avec un f. 146bis), ce dernier en occupe 17 (f. 140-156). Chaque feuillet mesure $26 \times 17 \mathrm{~cm}$, est réglé à la pointe sèche, et porte un nombre de lignes variables en minuscule caroline. Par ailleurs, il comporte 36 chapitres dont le texte dépend selon les cas de la version $Y$ et de la version $\mathrm{B}^{3}$. Seuls les treize premiers comportent des dessins à la plume rehaussés de vert, d'orange et de jaune, à l'exception du douzième, consacré au renard qui n'est pas assorti d'image. ${ }^{4}$ Curieusement, les illustrations sont de dimensions variables, contrairement aux réserves qui présentent des dimensions plus ou moins égales ensuite. Dans onze cas sur douze, une seule illustration accompagne le chapitre, celle 
relative au caladrius se continuant d'une page à l'autre. Par contre, la notice de la fourmi en comprend deux, nettement séparées par du texte.

2 Ce qui rend l'illustration de cet opuscule extrêmement intéressante est le fait qu'elle propose une mise en images interprétative du texte en ce qui concerne douze dessins sur treize $e^{5}$ - ce qui ne se trouve nulle part ailleurs dans cette proportion au sein de la tradition latine ${ }^{6}$. D'après Robert $\mathrm{G}$. Babcock, cette particularité s'expliquerait par le fait qu'à l'instar de la Psychomachie de Prudence pour laquelle c'est une certitude, le Physiologus aurait servi d'introduction à la lecture allégorique dans le but d'en enseigner l'exégèse. Et la nature des autres composantes du noyau ancien va effectivement dans ce sens. Quoi qu'il en soit, cette mise en images systématique des allégories ne se rencontre que dans le manuscrit byzantin Smyrneus B.8, aujourd'hui disparu, mais connu en grande partie par d'anciennes photos ${ }^{7}$. Mais alors même que ces deux manuscrits ont en commun le fait tout à fait exceptionnel de comporter une illustration évoquant à la fois les «natures» des animaux et leur interprétation allégorique, ils présentent un rapport texte-images totalement différent. En effet, autant l'illustration prend ses distances avec le texte (tout en collant à son esprit) dans l'exemplaire en grec, autant elle le met fidèlement en image dans le Bruxellensis. C'est donc de cet étonnant rapport de proximité qu'il va être question ici, ce qui sera l'occasion de mettre en évidence la manière dont le dessinateur a su doubler le texte tout en évitant une mise en images systématique qui aurait pu générer monotonie et ennui. Car, on le verra bientôt, l'illustration mêle croquis descriptifs, évocations des naturae et transpositions graphiques des allégories, avec une liberté confondante. Ainsi notre illustrateur préfère parfois représenter des personnages bibliques ou des pagani convoqués à un titre ou à un autre dans le chapitre, plutôt que le Christ ou le diable qui y interviennent comme pôles métaphoriques. Inversement, c'est l'aspect théologique qui domine parfois alors qu'ailleurs, c'est plutôt l'allégorisation morale, à moins que ce soient les naturae des animaux qui soient privilégiées. Il n'en reste pas moins que d'une manière générale, l'illustration colle au texte en reprenant l'essentiel des éléments narratifs et en mettant en image plusieurs métaphores et comparaisons signifiantes. Parallèlement, elle se caractérise encore par l'abondance de légendes identificatrices et explicatives, ainsi que par l'expressivité de la gestuelle prêtée aux différents protagonistes qui s'apparente à un véritable langage des signes. Pour toutes ces raisons, l'illustration du Bruxellensis acquiert un statut particulier qui l'affranchit en quelque sorte du texte tout en l'enrichissant.

\section{Le rapport Texte-Image dans les chapitres}

3 Voyons maintenant ces illustrations plus en détail ${ }^{8}$, dans leur rapport avec le texte, et suivant l'ordre des chapitres dans lesquelles apparaissent.

\section{LEO (f. 140r-140v)}

En l'occurrence, c'est dans la partie gauche du feuillet qu'a été concentrée l'évocation des naturae du lion puisqu'on y voit la mise en image de la première et de la troisième de ses natures. Ainsi, sous la représentation d'un chasseur cornant, accompagné du mot venator, on distingue ce qui doit être interprété comme un lion effaçant de sa queue les traces qu'il a laissées derrière lui, suivant la légende cooperit leo cum cauda vestigia sua 
résumant ce qui est dit dans le texte. En dessous, le lion ressuscite par son souffle son petit, sous le regard de la lionne. Dans ce cas, aucune légende n'accompagne le dessin qui d'ailleurs se comprend aisément sans l'aide de l'écrit. Seule la dimension temporelle y est évidemment absente : rien n'indique en effet que c'est le troisième jour après la naissance du lionceau qu'a lieu cette scène, alors qu'elle est primordiale dans l'association métaphorique avec la résurrection du Christ. Et aucune image de ce dernier ne vient le rappeler. Par contre, en haut à droite, est figurée la bénédiction par Jacob de Juda, ancêtre et préfigure de Christ, rappelée par la légende Benedicens Iacob filium. Sans doute l'illustrateur a-t-il choisi de représenter cette scène car l'évocation de Juda ouvre le chapitre et le clôt. En effet, on y lit d'abord : "Jacob bénissant son fils Juda, déclara "Juda, mon fils, petit du lion, qui le relèvera? » (Gen. 49,9), avant d'être à nouveau convoqué à propos de la résurrection du Christ : «Ainsi, le Père tout puissant ressuscita, au troisième jour d'entre les morts, son fils, notre Seigneur, comme le dit Jacob «Il a dormi comme le lion, et comme le petit du lion. Qui le réveillera?». (Gen. $49,9)$. On notera par ailleurs que la scène suggère un rapport de déférence affectueuse entre Juda et son père: ses genoux sont fléchis et ses mains sont tendues en signe d'acceptation et d'obéissance. Par ailleurs, la taille énorme de celles de Jacob, confère une importance particulière à son geste de bénédiction qui peut sans doute être étendu à la Tribu de Juda dont le Christ est issu.

\section{AUTALOPS (f. 140v-141r)}

Conformément au texte, on voit l'antilope essayant de dégager ses cornes prises dans des branches, alors qu'un homme lui transperce les flancs. La légende qui accompagne l'animal met en évidence la cause de ses problèmes Autalops firmavit (?) cornua sua in arborem. Et celle qui accompagne l'homme identifié comme chasseur - venator introduit en quelque sorte la morale de l'histoire, synthétisée par la légende exprimée sur le mode direct Homo dei abscide omnia vitia mala a te ut ne comprehenderis a diabolo qui est assortie d'une scène dans laquelle le Christ s'adresse à un homme visiblement réceptif, comme en témoignent ses paumes ouvertes ${ }^{9}$. Dans ce cas, la légende s'apparente à un fragment de discours divin, transformant l'exemplum en authentique parabole, comme on le voit aussi dans le chapitre de l'aigle ${ }^{10}$. Ni dans la légende ni dans le dessin, il n'est expressément fait allusion aux deux principales causes des vices incriminés : le vin et les femmes, suivant la citation de l'Ecclesiaste qui clôt le chapitre : «Vinum enim et mulieres apostizare faciunt homines a deo ».

\section{LAPIDES IGNIFERI (f. 141v)}

On reste dans un même ordre d'idées dans le chapitre suivant consacré aux pierres ignifères, symbolisant évidemment l'attraction fatale entre hommes et femmes brûlant de passion l'un pour l'autre. L'illustration ${ }^{11}$ suit fidèlement le texte. On voit en haut à gauche, une femme poussée par l'Ange de Satan qui présente à un homme la pierre mâle et la pierre femelle qui, rapprochées l'une de l'autre, commencent à s'enflammer. À l'évidence, l'homme en accepte le don, la main droite levée et grande ouverte. Dans le registre du dessous, la Chaste Suzanne (Dan.), identifiée par son prénom (Susanna), fait face à deux hommes dont on se serait attendu à ce qu'ils soient présentés comme les vieillards libidineux, mais qui le sont en tant que falsi presbiteri en écho au texte qui met les homines Dei en garde contre la proximité des femmes, "afin que le feu ne les 
embrase pas et ne consume tous les biens que le Christ leur a apportés ", pour paraphraser le Physiologue. Le début de la légende, malheureusement effacée, évoquait sûrement leurs avances, comme le font leurs gestes destinés tant à convaincre qu'à menacer. Ceux de Suzanne, paumes des mains tournées vers l'extérieur, expriment clairement le refus. La scène suivante évoque le péché originel, Ève et Adam étant accompagnés de leur nom ( $E v a /$ Adam). On a ainsi, en diptyque, deux femmes tentées dont l'une «consentit à la Chute ", tandis que l'autre "vainquit en observant la Loi ». Curieusement il n'est pas question dans l'illustration de Joseph et de Samson, autres figures exemplatives dont il est question dans le texte. Sans doute ce choix s'explique-til par la teneur de la conclusion : «Pour cette raison, il faut se protéger des femmes par les commandements divins, parce que leur péché qui fait perdre l'esprit à ceux qui obéissent, a commencé dès le commencement, c'est-à-dire depuis Adam jusqu'à ses fils ».

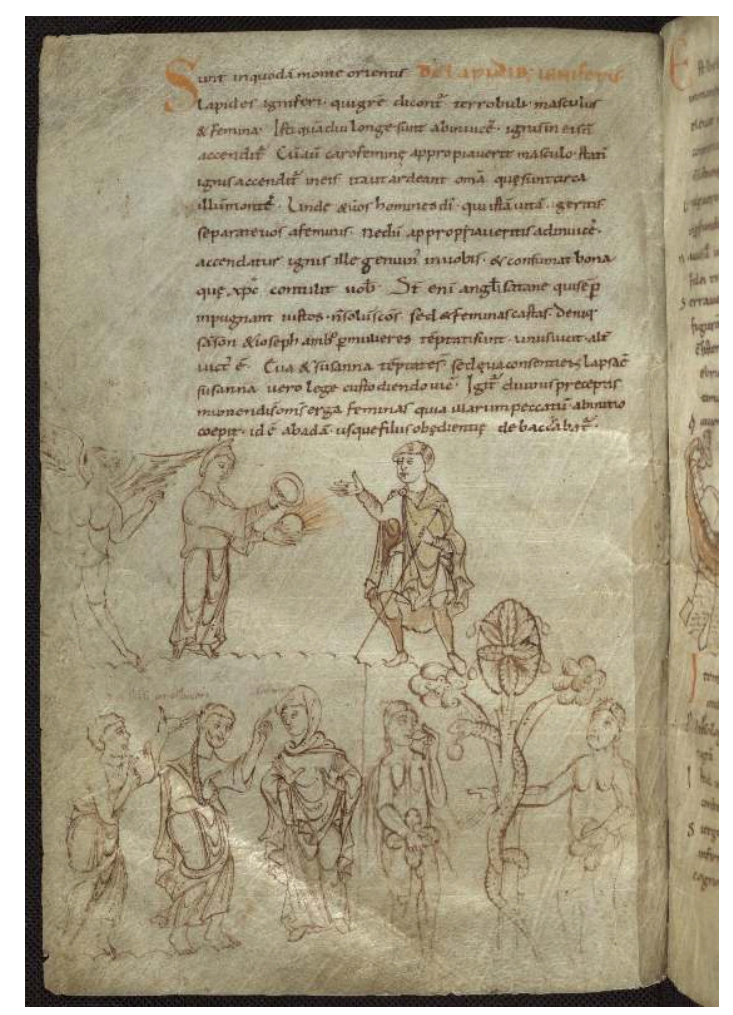

Fig. 1. Lapides igniferi. ms. Bruxelles, KBR, 10066-77, f. 141v. (Copyright Bibliothèque royale de Belgique, Bruxelles)

\section{CALADRIUS (f. 142v-143r)}

$7 \quad$ L'illustration du chapitre consacré au caladrius se poursuit d'une page à l'autre ${ }^{12}$. Le premier croquis, en bas à gauche, fait écho à son habitat dans les "Palais des Rois». C'est en effet dans cet endroit qu'un homme est en train de le capturer avec un filet: Ubi caladrius comprehenditur in domibus regum. Les deux scènes suivantes évoquent sa capacité à prévoir l'issue d'une maladie. Dans le premier cas, le caladre détourne les yeux du malade Ubi calidrius evertit oculos suos ab infirmo, prédisant ainsi une issue fatale - ce qui explique l'attitude désespérée de l'homme qui se trouve à la tête du lit. Dans le second cas, l'oiseau a été figuré fixant du regard le malade, puis s'envolant vers le soleil personnifié, à l'intérieur de l'astre ardent. Une légende supplée à ce qui ne peut être 
rendu graphiquement Ubi caladrius aspicit egrum et sanatur, et portans infirmitatem suam a radio solis. Affirmation sur laquelle est évidemment construite la deuxième partie de la métaphore christique résumée par la légende Ipse tulit iniquitates nostras qui accompagne la représentation du Sauveur, bras écartés. Par contre, dans l'illustration, il n'est guère fait allusion à celle qui est construite sur la première nature du caladre, et qui apparaît seulement dans le texte, à savoir que le Christ « a détourné son visage des Juifs à cause de leur manque de foi, avant de se tourner vers notre peuple et de porter ses infirmités et ses péchés ». Notons encore que le personnage qui a la main sur le front du malade exprime sa joie de le voir guéri par un grand geste de tout le bras.

8 Le croquis se développant dans la marge fait écho au dernier paragraphe qui lui-même revient sur une affirmation faite tout au début, à savoir que le caladrius est un animal impur selon la Bible, et qu'on ne peut donc en consommer. En effet, suivant en cela la version $B$, on lit en conclusion tout un petit développement sur l'ambivalence de plusieurs animaux - serpent, lion, aigle - qui explique que le caladre puisse être à la fois impur et vertueux, contrairement à ce qu'on pourrait croire à première vue. À l'appui de la démonstration, est notamment rappelé l'épisode du Serpent d'airain à travers la célèbre phrase de Jean : "Car tout comme Moïse éleva le serpent dans le désert, il est nécessaire que de la même manière le Fils de l'Homme soit élevé » (Ioh. 3.14) - ce qui justifie la représentation de cette scène, accompagnée de la légende explicative Ubi Moyses exaltauit serpentem in [deser]to. On reconnaît Moïse, tenant un livre ouvert d'une main, et de l'autre faisant un geste explicatif devant un groupe d'Hébreux - outre ce qui reste du dessin comportant le serpent. Peut-être sa présence inaugure-t-elle la longue série de Serpents d'airain figurés dans l'art roman de la région de la Meuse, et qui en constitue presque une "marque de fabrique ». En tout cas, cela renforce l'hypothèse d'une origine ${ }^{13}$ mosane et sans doute liégeoise du manuscrit.

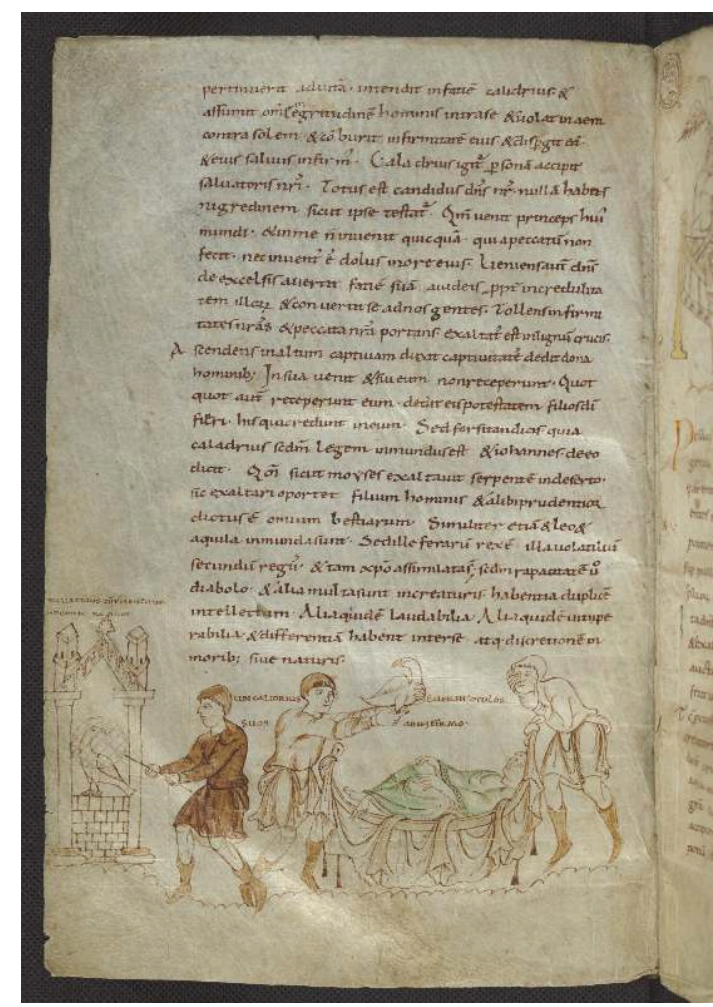

Fig. 2a-b. Caladrius. ms. Bruxelles, KBR, 10066-77, f. 142v et 143r. (Copyright Bibliothèque royale de Belgique, Bruxelles) 


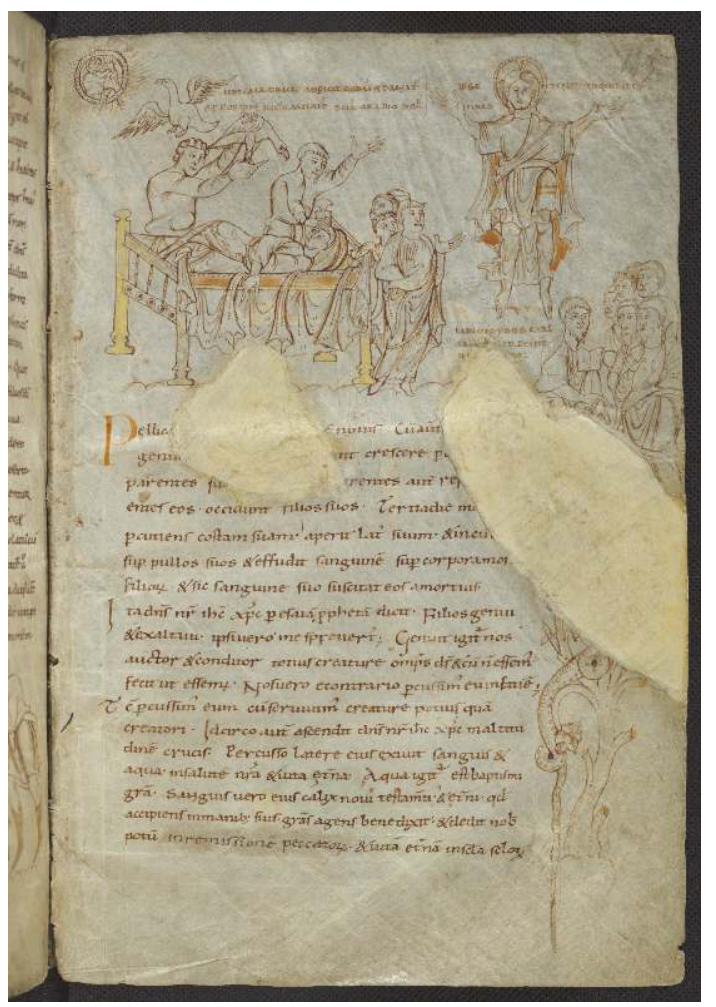

Fig. 2a-b. Caladrius. ms. Bruxelles, KBR, 10066-77, f. 142v et 143r. (Copyright Bibliothèque royale de Belgique, Bruxelles)

\section{PELICANUS (f. 143r-143v)}

9 Nous ne nous attarderons pas longtemps sur le chapitre relatif au pélican, dans la mesure où plus que jamais, l'illustration y apparaît comme la transposition fidèle des principaux éléments narratifs et allégoriques. On y voit au-dessus, successivement, un jeune pélican frappant un de ses parents, accompagné de la légende Pellicani interficiunt parentes suos, puis la mère versant son sang sur deux petits, les arrachant ainsi à la mort, comme le rappelle l'inscription: Ubi pellicana aperit costam suam et effudit sanguinem suum super filios suos, et suscitat eos a mortuis. Le registre principal est occupé par une Crucifixion dont tous les protagonistes sont nommés (Beata Maria / Iesus Nazarenus / Sanctus Johannes / Longinus / Steph[aton]) - ce qui ne pouvait évidemment mieux convenir à cette place. Les mots comme les images rappellent ainsi que les hommes comme les oisillons sont les bénéficiaires du sacrifice qui a été consenti pour eux.

\section{NYCTICORAX (f. 143v-144r)}

Dans le chapitre sur le nycticorax, c'est la citation liminaire du roi psalmise «Je suis comme le hibou dans sa demeure » (Ps. CI.7) qui est reprise dans la légende surmontant la scène du registre supérieur Factus sum sicut nicticorax in domicilio. Celle-ci comprend un groupe d'hommes s'avançant vers un souverain couronné et nommé Cesar, assis sous un portique. Curieusement, c'est sur celui-ci qu'est perché notre oiseau dont rien n'indique par ailleurs qu'il est un animal nocturne. Par contre, la comparaison qui est bâtie sur ce comportement est fidèlement mise en image. En effet, d'une part on lit: 
«[Le nycticorax] porte la ressemblance du peuple des Juifs qui, lorsque le Seigneur notre Sauveur est venu le sauver, le repoussèrent et dirent : 'Nous n'avons pas d'autre roi que César : celui-ci nous ne savons pas qui il est' ». Et d'autre part, on voit un groupe de Juifs (IudaĘi) se détournant du Christ et faisant part de leur allégeance à César, comme l'indique doublement la légende Nos regem non habemus nisi Caesarem, ainsi qu'une gestuelle particulièrement expressive. De son côté, le Christ les exhorte à rendre à César ce qui appartient à César Reddite quae sunt Caesaris Caesari, ce qui n'est pas rappelé dans le texte et qui constitue donc un apport intéressant.

\section{AQUILA (f. 144r-144v)}

11 En ce qui concerne le chapitre consacré à l'aigle, la transposition graphique est à nouveau extrêmement fidèle. À gauche, on voit tout ce qui le concerne; à droite, la leçon destinée aux hommes qui en est tirée. Ainsi, au-dessus, l'oiseau est figuré en train de brûler ses ailes vieillies aux rayons du soleil représenté personnifié - ce que confirme la légende Ubi aquila comburit alas suas ad radios solis. En dessous, on le découvre au centre de ce qui doit être compris comme la source ou la fontaine dans laquelle il se plonge trois fois Ubi trina vice se fonte mergit. Et à côté, il apparait tout rajeuni, dans les branches d'un arbre Ubi aquila renovata sede in arbore. Toutes les étapes de la cure de jouvence sont donc mises en image! Dans la partie droite, le Christ exhorte les hommes à se renouveler, dans une forme proche de celle qu'on trouve dans le texte : Et tu homo, sive Iudeus, sive Christianus, renova te sicut aquila iuventutem suam ! On peut peut-être reconnaître le chrétien dans l'homme qui tend les mains dans un geste d'acceptation, et le Juif dans celui qui semble faire un geste de refus. Le moyen de parvenir à cette rénovation dont l'aigle montre l'exemple - le baptême - est évoqué endessous, comme le rappelle l'inscription Ubi renovatur homo per babtismum sicut aquila. Ici, c'est la légende qui fait le lien entre la nature de l'oiseau et la régénération sacramentelle, car la référence au baptême n'est pas explicite dans le texte. En effet, il n'y est fait allusion qu'au travers d'une citation de l'évangile de Jean : «Celui qui n'est pas né à nouveau au nom du Père, du Fils et du Saint-Esprit, ne peut pas pénétrer dans le royaume de Dieu » (Ioh. 3,5). 


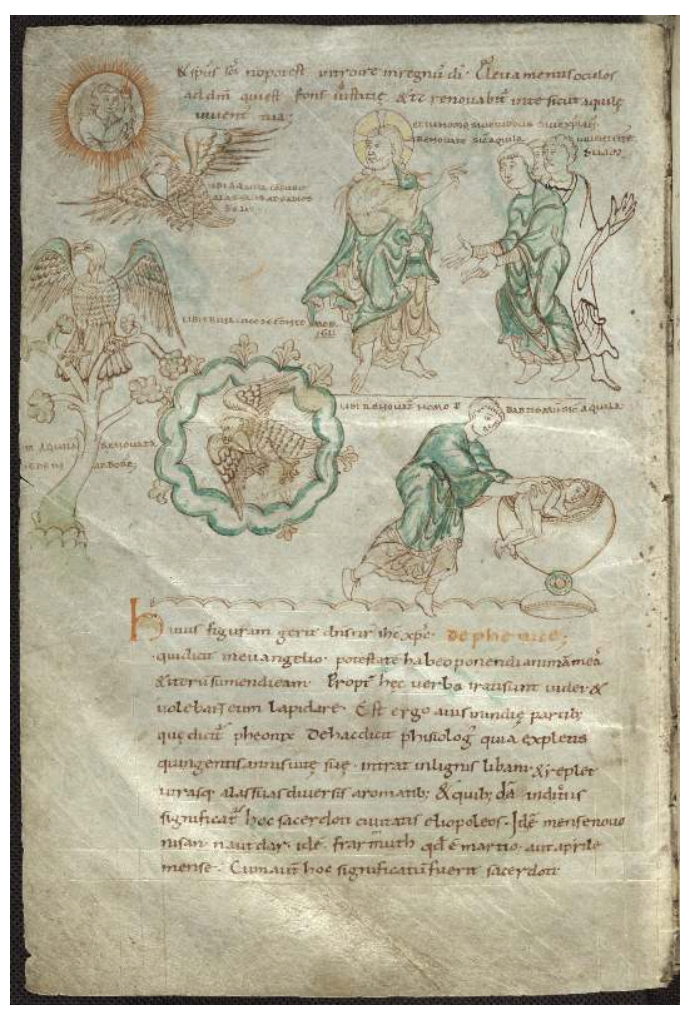

Fig. 3. Aquila. ms. Bruxelles, KBR, 10066-77, f. 144v (Copyright Bibliothèque royale de Belgique, Bruxelles)

\section{PHOENIX (f. 144v-145r)}

On en arrive au chapitre consacré au phénix, qui d'emblée proclame qu'«il porte la ressemblance de notre Seigneur Jésus-Christ qui a dit dans l'Evangile : 'J'ai le pouvoir de donner mon âme et de la reprendre à nouveau' ». Suit la description des naturae de l'oiseau dont on trouve un écho simplifié dans l'illustration. On le voit d'abord perché sur un cèdre du Liban (Phonix / Cedrus Libani), comme précisé ; puis ressuscité sur l'autel $\mathrm{du}$ temple d'Héliopolis où le découvre le prêtre, ainsi que le précise la légende Ubi sacerdos invenit renovatam aviculam super aram templi. Curieusement, ce dernier l'entoure de ses grandes mains (comme le prêtre saisissant le calice ?) - ce qui confère à la scène un caractère intimiste qui pourrait paraître déconcertant dans ce contexte. Plus loin, un Christ immense s'adresse à la foule, deux doigts relevés dans le geste de l'enseignant. La scène est accompagnée de l'inscription Ubi dominus dicit: "Non veni solvere legem sed adimplere » (Matth. 5.17) : «Je ne suis pas venu abroger la Loi mais la compléter " - référence au Sermon sur la montagne dont il est aussi question dans le texte à propos des deux Testaments assimilés aux ailes de l'aigle. Par contre, l'attitude colérique prêtée aux Juifs face au mystère de la résurrection qui y est aussi évoqué, n'est guère manifeste dans l'illustration. Celle-ci est donc en partie sélective, comme souvent.

\section{FORMICA (f. 145v-146r)}

La manière dont a été conçue l'illustration du chapitre consacré à la fourmi est plus déconcertante. En effet, comme on l'a dit, elle se subdivise en trois parties séparées par 
du texte, et s'étale sur deux pages - ce qui ne se rencontre nulle part ailleurs. En outre, elle se concentre uniquement sur les naturae des insectes, si l'on excepte la transposition graphique de l'exhortation du roi Salomon « Va vers la fourmi, paresseux, et imite-la!» (Proverbes 6,6) qui s'étale en première ligne. Ainsi voit-on le souverain assis sur une chaise curule, identifié par l'inscription Salomon rex, face à un homme à moitié endormi qui n'est autre qu'un paresseux auquel il s'adresse. Seuls toutefois sont conservés les premiers mots de l'interpellation, soit 0 piger suivis de signes de rasure. À sa gauche sont dessinées des fourmis grimpant le long d'épis de blé, ainsi qu'une fourmilière. Les inscriptions rappellent successivement qu'elles en portent et les rassemblent, sous-entendu, dans leur demeure Ubi triticum de spicis tollunt / Ubi formice triticum congregant. La comparaison avec les Vierges sages, elles aussi prévoyantes, n'est pas illustrée.

14 La deuxième nature de la fourmi concerne son habitude de fendre en deux les grains qu'elle a emmagasinés pour éviter qu'ils pourrissent, ce qui, dit le texte, doit inciter l'homme à distinguer les choses de l'esprit et celles du corps, pour éviter que la lettre ne le tue. Curieusement, alors qu'on ne s'attendait guère à trouver d'illustration eu égard à l'impossibilité de mettre ces propos en images, à moins d'y évoquer les Juifs que la lettre a tués (comme dit dans le texte), il y a à nouveau un croquis rassemblant épis de blé, rangée de fourmis et fourmilière - sans légende, cette fois. Peut-être est-ce une partie inachevée?

Quant au dessin illustrant la troisième nature de la fourmi, il diffère finalement peu des deux précédents, si ce n'est qu'à côté des épis de blé se trouvent des épis plus petits que le texte et la légende présentent comme ceux de l'orge. Ce sont ces épis-là que la fourmi dédaigne pour ne prendre que ceux du blé, de meilleure qualité. Le chapitre se clôt sur une comparaison entre l'orge, "la nourriture des bêtes sans intelligence", et la doctrine des hérétiques qu'il faut fuir. Hélas, ni Sabellius, ni Mani ni les autres n'ont été représentés, nous privant d'une belle galerie d'hérétiques notoires. À leur place, on ne trouve donc que des épis d'orge que les fourmis traversent et des épis de blés sur lesquels elles grimpent, comme il est dit dans la légende Ubi formice transerunt ordea ${ }^{\text {scorr. }}$ suscrite] et veniunt ad triticum et ascendunt in sumitatem spici. Quant à la fourmilière qui est dessinée à gauche, elle est accompagnée d'une légende, en partie redondante, puisqu'elle rappelle que les fourmis y rassemblent leur nourriture, et en partie originale, puisqu'elle précise que la fourmilière est construite à l'aide de brindilles : Ubi formice domum fecerunt ex diversis lignis et congegraverunt diversa ciborum.

Dans le cas de ce chapitre, le rapport texte-images est vraiment très particulier puisque la partie éthologique a été fortement privilégiée au détriment de la mise en images des leçons morales et théologiques. Il est malaisé de justifier ce choix.

\section{SIRENAE ET ONOCENTAURI (f. 146r-146v)}

Comme dans le texte où sirènes et onocentaures sont évoqués successivement, on retrouve ces deux créatures sur deux registres qui se suivent, au niveau de l'illustration ${ }^{14}$. Une sirène y apparaît jouant d'un instrument à corde, dans l'intention d'abuser des hommes, comme le précise la légende Ubi syrenae musicam sonant ad decipiendos homines qui synthétise la première partie du texte. La seconde est illustrée par deux sirènes déchirant le corps d'un homme déjà mort, comme indiqué Ubi dilaniant eos jam mortuos. Les hommes attirés par tous les plaisirs détaillés dans le chapitre et qui 
en perdent toute vigueur d'esprit, à l'instar des victimes des sirènes, n'apparaissent guère dans l'illustration.

Pour ce qui est de l'onocentaure, uniquement présenté comme un hybride d'homme et d'âne, les choses sont un peu différentes. Surmonté du mot Onocentaurus, il apparaît à gauche, en train de transpercer un lièvre, alors que deux hommes discutent de manière véhémente, dans la partie droite de la page. L'un d'eux est un bilinguis dont la duplicité l'apparente au centaure à la double nature, et qui tente de tromper son interlocuteur, comme en témoigne la légende Ubi bilinguis diversis modis fallitur. Sans doute le pouvoir est-il au centre des enjeux, puisque le mot Potestas est écrit entre eux. On notera qu'il n'est nullement question d'une problématique liée au pouvoir dans le texte. Il s'agit donc d'un ajout du dessinateur.

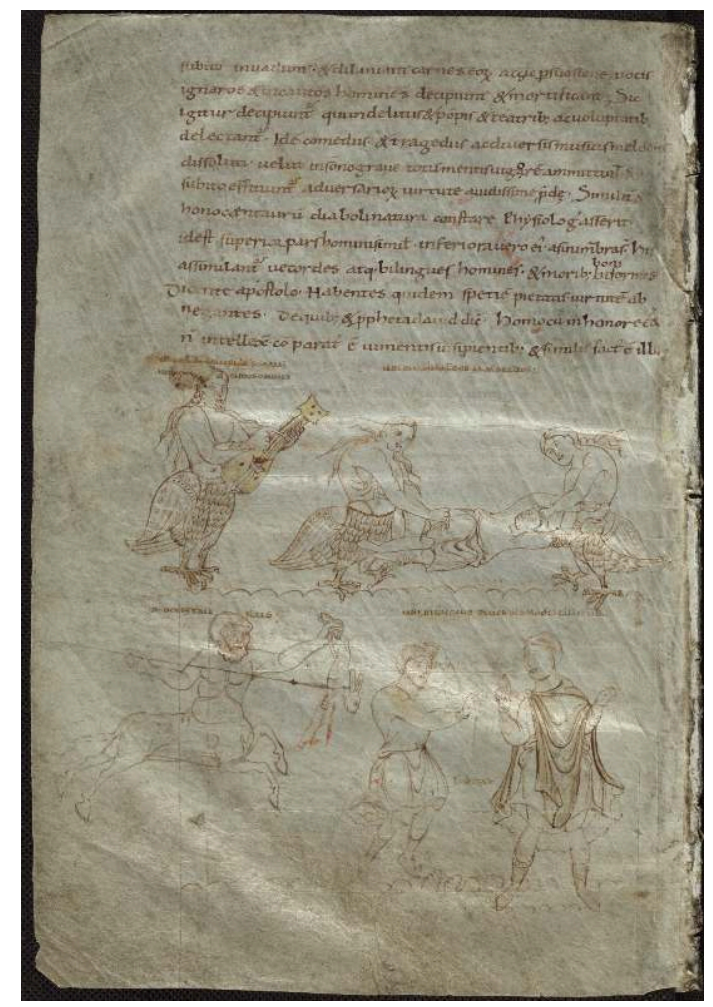

Fig. 4. Sirenae et Onocentauri. ms. Bruxelles, KBR, 10066-77, f. 146v. (Copyright Bibliothèque royale de Belgique, Bruxelles)

\section{UNICORNIS (f. [146/146bis]-147r)}

La dernière illustration qui a été réalisée concerne l'unicorne dont on voit la capture selon les modalités bien connues, dans la partie supérieure de l'image. À cet égard, on notera surtout la manière originale dont elles s'expriment graphiquement. D'une part, la jeune vierge semble décider l'unicorne à la suivre en la prenant doucement par le menton, d'autre part, le serviteur du roi amène cette dernière par la main vers le palais - ce qui donne une belle chaîne humaine et animale! Les différentes scènes ne sont pas accompagnées d'inscriptions, et correspondent à la première partie du texte pour l'essentiel - à savoir que l'unicorne ne peut être capturée que par une jeune vierge. Cette dernière figure encore, en dessous à droite, assise sur un siège curule, tenant l'animal avec tendresse, alors qu'il pose son museau sur ses genoux, corne inclinée vers l'arrière. Il a été dessiné plus petit que sur le registre du haut, comme pour mieux 
signifier que sa taille menue signifie « l'humilité de l'Incarnation, à propos de laquelle Il [le Seigneur a] dit 'mettez-vous à mon école car je suis doux et humble de cœur' » (Matth. 11, 29), comme on le lit dans le texte et dans la légende Discite a me quoniam mitis sum et humilis corde qui accompagne la scène de gauche dans laquelle le christ enseignant s'adresse à deux disciples dont l'attitude exprime l'assentiment.

Mais au-delà de ces particularités, un des intérêts de ce dessin est de nous apprendre que le chapitre qu'il illustre dépend d'une version différente de celle qui a été utilisée dans le texte du feuillet 146 bis qui a remplacé celui d'origine, à la fin du XII ${ }^{\mathrm{e}}$ siècle $^{15}$. En effet, des détails comme la présence dans l'image du palais et de son hôte royal renvoient plutôt à la version $\mathrm{B}^{16}$ ([...] et sic comprehenditur et exhibetur in palatio regis), alors qu'il n'en est pas question dans le passage correspondant du feuillet de remplacement qui dépend d'une variante de la version $Y$.

Quoi qu'il en soit de ce dernier cas dans lequel le rapport Texte-Image est impossible à apprécier dans le détail, celui-ci est à la fois proche et original au niveau des autres chapitres. De fait, l'illustration, accompagnée le plus souvent de nombreuses légendes, se présente presque comme son double. Il n'en reste pas moins que la transposition graphique du texte n'a rien de mécanique et que chacun des deux moyens d'expression conserve sa spécificité tout en contribuant, chacun à sa manière, à la fixation de la leçon de théologie ou de morale. Quant à la présence même des inscriptions qui servent à la fois à identifier les scènes et leurs protagonistes et à remplir l'espace, elles annoncent celles qui caractériseront les œuvres d'orfévrerie mosane d'époque romane. Ajoutées au fait que ce manuscrit est le seul Physiologus latin dont l'iconographie soit en partie fondée sur la pensée typologique, dont on sait qu'elle s'est développée sous l'influence de centres d'études théologiques et d'exégèse biblique comme Liège ${ }^{17}$, on ne peut plus guère mettre en doute son origine mosane et sans doute vraisemblablement liégeoise, comme le suggère Robert Babcock de manière convaincante, en s'appuyant sur d'autres arguments. L'évocation de l'épisode du Serpent d'airain dans l'illustration pourrait même constituer la plus ancienne représentation connue d'une préfigure ${ }^{18}$ qui connut une faveur exceptionnelle dans l'art de cette région et dans son aire d'influence ${ }^{19}$.

\section{BIBLIOGRAPHIE}

BABсоск R.G., The Psychomachia Codex from Saint Lawrence (Bruxellensis 10066-77) and the Schools of Liège in the Tenth and Eleventh Centuries, Turnhout, Brepols (Bibliologia, 42), 2017. BAXTER, R., Bestiaries and their Users in the Middle Ages, London, Sutton Publishing ; Courtauld Institute, 1998.

BAXTER R., « Learning from Nature : Lessons in Virtue and Vice in the Physiologus and Bestiaries ", in Virtue and Vice : the Personifications in the Index of Christian art, HOURIHANE C. (éd.), Princeton, Princeton University (Index of Christian art. Resources Series, 1), 2000, p. 21-41. 
BERNABÒ M., avec la coll. de PEERS M. G. et TARASCONI, R., Il Fisiologo di Smirne. Le miniature del perduto codice B. 8 della Biblioteca della Scuola Evangelica di Smirne, Firenze, SISMEL, edizioni del Galluzzo (Millennio medievale, 7), 1998.

CARMODY F. J., Physiologus latinus. Versio B, Paris, 1939

CARMODY F. J., « Physiologus latinus Versio Y », University of California Publications in Classical Philology, 12 (7), 1941, p. 95-134.

DESCATOIRE Ch., «Figures iconographiques : images de la Croix, correspondances typologiques, allégories et vertus », in Une Renaissance. L'art entre Flandre et Champagne 1150-1250, Paris, 2013 (Catalogue d'exposition).

DOROFEEVA A., « Miscellanies, Christian reform and early medieval encyclopaedism : A reconsideration of the pre-bestiary Latin Physiologus manuscripts ", in Historical Research, 90, $n^{\circ} 250$ (nov. 2017), p. 665-682.

GARNIER Fr., Le langage de l'image au Moyen Âge. 1. Signification et symbolique, Paris, Le Léopard d'or, 1982.

HENRIET P., « Relire l'autel de Stavelot », in Orfèvrerie septentrionale. XII ${ }^{\mathrm{e}}$ et XIII ${ }^{\mathrm{e}}$ s. L'œuvre de la Meuse II, George, Ph., éd., Liège, Trésor de la Cathédrale (Feuillets de la Cathédrale de Liège), 2016, p. 179-208: 186-187.

HOMBURger O., vON STEIGER Ch., Physiologus Bernensis, Voll-faksimile Ausgabe des Codex Bongarsianus 318 der Bürgerbibliothek, Bern-Basel, 1964.

LECLERCQ-MARX J., « Le manuscrit B. R. 10074. Physiologus de naturis animalium et bestiarum », in Annales du XLIV ${ }^{\mathrm{e}}$ Congrès de la Fédération des Cercles d'Archéologie et d'Histoire de Belgique (Huy 1976), Bruxelles, t. 3, 1980, p. 790-795

LECLERCQ-MARX J., La Sirène dans la pensée et dans l'art de l'Antiquité et du Moyen Âge. Du mythe païen au symbole chrétien, Bruxelles, Académie royale de Belgique (Publications de la Classe des Beaux-Arts. Coll. In- $4^{\circ}, 3^{\text {e }}$ série, t. 2), 1997, p. 72-74 et ill. 39. Version libre numérisée sur le site : http://www.koregos.org/fr/jacqueline-leclercq-marx_la-sirene-dans-la-pensee-et-dans-l-art-del-antiquite-et-du-moyen-age/4389/

LECLERCQ-MARX J., « La sirène et l'onocentaure dans le Physiologus grec et latin, et dans quelques Bestiaires. Le texte et l'image ", in Bestiaires médiévaux. Nouvelles perspectives sur les manuscrits et les traditions textuelles, Actes $\mathrm{du} \mathrm{XV}^{\mathrm{e}}$ colloque de la Société internationale Renardienne, Louvain-la-Neuve, 19-22 août 2003, Van den Abeele, B. (éd.), Louvain-la-Neuve, Université Catholique de Louvain (Publications de l'Institut d'Études médiévales. Textes, Études, Congrès, 21), 2005, p. 169-182.

LECLERCQ-MARX J., « Drôles d'oiseaux. Le caladre, le phénix, la sirène, le griffon et la serre dans le Physiologus, les Bestiaires et les encyclopédies du XIII ${ }^{\mathrm{e}}$ siècle. Mise en perspective ", in Déduits d'oiseaux au Moyen Âge, Actes du $32^{\text {e }}$ Colloque du CUER MA, Aix-en-Provence, 22-24 mars 2007, Ch. Connochie-Bourgne (éd.), Sénéfiance, 54, 2009, p. 163-178, 302-306.

LECLERCQ-MARX J., « L'illustration du Physiologus grec et latin, entre littéralité et réinterprétation de l'allégorie textuelle. Le cas des manuscrits Bruxellensis 10066-77 et Smyrneus B.8 ", in L'allégorie dans l'art du Moyen Âge. Formes et fonctions. Héritages, créations, mutations, HECK Ch. (ed.), Turnhout, Brepols (Les Études du RILMA, 2), 2011, p. 141-155.

MARESCHAL V., Un témoin latin illustré du Xe siècle du Physiologus. Le Bruxellensis 10066-77, Louvain-la-Neuve, UCL, 1997 (mémoire de fin d'études). 
NAGATOMO M. «Bruxelles Physiologus. A Study on the Formation of Illustrations ", in Lux Artium, Tokyo, Cyuokoron Bijutsu Shuppan, 2010, p. 34-48 (en japonais).

\section{NOTES}

1. On se réfèrera à ВАВСоск, R.G., The Psychomachia Codex from Saint Lawrence (Bruxellensis 10066-77) and the Schools of Liège in the Tenth and Eleventh Centuries, Turnhout, Brepols (Bibliologia, 42), 2017 : 28-32 pour en connaître la liste la plus complète.

2. La partie Physiologus n'a jamais fait l'objet d'une étude monographique de quelqu'ampleur, si ce n'est sous la forme d'un mémoire de fin d'études d'histoire, présenté à l'Université Catholique de Louvain s. dir. de B. Van den Abeele (MARESCHAL V., Un témoin latin illustré du Xe siècle du Physiologus. Le Bruxellensis 10066-77, Louvain-la-Neuve, UCL, 1997), auquel j'emprunte essentiellement la transcription (vérifiée ici) de certains passages ainsi que des inscriptions apparaissant dans le champ des illustrations. Toutefois ВАВсоск, The Psychomachia Codex, tout récemment publié, constitue une importante contribution à la connaissance des deux textes majeurs du noyau le plus ancien du recueil. À cet égard, le chapitre 6 est exclusivement consacré au Physiologus (p. 201-226), mais il en est encore question dans les chapitres 2 et 7 . Du point de vue spécifique de l'illustration, nous nous permettons de renvoyer à notre brève étude iconographique " Le manuscrit B. R. 10074. Physiologus de naturis animalium et bestiarum », in Annales du XLIV Congrès de la Fédération des Cercles d'Archéologie et d'Histoire de Belgique (Huy 1976), Bruxelles, t. 3, 1980, p. 790-795, et surtout à «L'illustration du Physiologus grec et latin, entre littéralité et réinterprétation de l'allégorie textuelle. Le cas des manuscrits Bruxellensis 10066-77 et Smyrneus B. 8 ", in L'allégorie dans l'art du Moyen Âge. Formes et fonctions. Héritages, créations, mutations, dir. HECK Ch., Turnhout, Brepols (Les Études du RILMA, 2), 2011, p. 141-155, dont le propos croise en partie celui du présent article, mais suivant une autre approche. On ajoutera à la bibliographie qui l'accompagne les références suivantes: BAXTER, R., Bestiaries and their Users in the Middle Ages, London, Sutton Publishing; Courtauld Institute, 1998, essentiellement p. 62-74, et p. 82-83 ; DOROFEEVA A., "Miscellanies, Christian reform and early medieval encyclopaedism: A reconsideration of the pre-bestiary Latin Physiologus manuscripts ", in Historical Research, vol. 90, $\mathrm{n}^{\circ} 250$ (nov. 2017), p. 665-682, ainsi que nAGATomo M. « Bruxelles Physiologus. A Study on the Formation of Illustrations ", in Lux Artium, Tokyo, Cyuokoron Bijutsu Shuppan, 2010, p. 34-48 (en japonais). À noter qu'Anna Dorofeeva prépare actuellement un ouvrage sur le Physiologus latin dont un chapitre sera consacré au Bruxellensis 10066-77.

3. Ces deux versions ont été éditées par F. J. CARMODY. Voir Physiologus latinus. Versio B, Paris, 1939 et «Physiologus latinus Versio Y », University of California Publications in Classical Philology, 12, 7 (1941), p. 95-134.

4. Le manuscrit est reproduit intégralement en version numérique sur le site belgica.kbr.be.

5. Le dessin illustrant le chapitre de la serre l'évoque uniquement en sa qualité de res prima. Il n'en sera donc pas question ici.

6. Seul le premier des quatre tableaux qui accompagnent la notice consacrée au lion, dans le Physiologus carolingien de Bern (Bürgerbibliothek, lat. 318), fait écho à la relation suggérée métaphoriquement dans le texte, entre l'animal et le Christ. Voir HOMBURGER O., VON STEIGER Ch., Physiologus Bernensis, Voll-faksimile Ausgabe des Codex Bongarsianus 318 der Bürgerbibliothek, BernBasel, 1964, sur base du ms. Bern, Bürgerbibliothek, lat. 318 (IX ${ }^{\mathrm{e}}$ s.).

7. Ce manuscrit date de la fin du $\mathrm{XI}^{\mathrm{e}}$ siècle ou serait plus vraisemblablement une copie d'un exemplaire de cette époque, exécutée un peu après le milieu du XIV ${ }^{\mathrm{e}}$ siècle. En tout cas, c'est la datation adoptée par Massimo Bernabò à qui l'on doit l'étude la plus complète et la plus récente sur le sujet. Voir BERNABò M., avec la coll. de PEERS M. G. et TARASCONI, R., Il Fisiologo di Smirne. Le 
miniature del perduto codice B. 8 della Biblioteca della Scuola Evangelica di Smirne, Firenze, SISMEL, edizioni del Galluzzo (Millennio medievale, 7), 1998.

8. On ne renvoie ci-après qu'aux études dans lesquelles les illustrations sont reproduites et commentées ou tout au moins, mises en contexte.

9. GARNIER Fr., Le langage de l'image au Moyen Âge. 1. Signification et symbolique, Paris, Le Léopard d'or, 1982, p. 161-164.

10. Voir infra, passim.

11. Commentaire de cette image dans BAXTER R., «Learning from Nature : Lessons in Virtue and Vice in the Physiologus and Bestiaries", in Virtue and Vice: the Personifications in the Index of Christian art, éd. Hourihane, C., Princeton, Princeton University Press (Index of Christian art. Resources Series, 1), 2000, p. 36-38 et fig. 3.

12. LECLERCQ-MARX J., « Drôles d'oiseaux. Le caladre, le phénix, la sirène, le griffon et la serre dans le Physiologus, les Bestiaires et les encyclopédies du XIII ${ }^{\mathrm{e}}$ siècle. Mise en perspective ", in Déduits d'oiseaux au Moyen Âge, Actes du 32 ${ }^{\mathrm{e}}$ Colloque du CUER MA, Aix-en-Provence, 22-24 mars 2007, Ch. Connochie-Bourgne (éd.), Sénéfiance, 54, 2009, p. 163-178: 166 (illustrations p. 302-306). Sur l'image de la serra (f. 142r), voir note 5.

13. Voir infra, passim.

14. Voir LECLERCQ-MARX, J. « La sirène et l'onocentaure dans le Physiologus grec et latin, et dans quelques Bestiaires. Le texte et l'image", in Bestiaires médiévaux. Nouvelles perspectives sur les manuscrits et les traditions textuelles, Actes $\mathrm{du} \mathrm{XV}^{\mathrm{e}}$ colloque de la Société internationale Renardienne, Louvain-la-Neuve, 19-22 août 2003, Van den Abeele, B. éd., Louvain-la-Neuve, Université Catholique de Louvain (Publications de l'Institut d'Études médiévales. Textes, Études, Congrès, 21), 2005, p. 169-182 (fig. 51-56) : 172-173 et fig. 51, ainsi que Ead., La Sirène dans la pensée et dans l'art de l'Antiquité et du Moyen Âge. Du mythe païen au symbole chrétien, Bruxelles, Académie royale de Belgique (Publications de la Classe des Beaux-Arts. Coll. In- $4^{\circ}, 3^{\text {e }}$ série, t. 2), 1997, p. 72-74 et ill. 39. Version libre numérisée sur le site HTTP://WWW.KOREGOS.ORG/FR/JACQUELINE-LECLERCQMARX_LA-SIRENE-DANS-LA-PENSEE-ET-DANS-L-ART-DE-L-ANTIQUITE-ET-DU-MOYEN-AGE/4389/

15. Suivant BABCOCK, The Psychomachia Codex, p. 213-215 et pl. 9.

16. Voire, à la version B-Is, en ce qui concernerait la manière dont l'unicorne, cessant d'être agressive, vient loger son museau dans le sein de la vierge. Voir Loc. cit.

17. Comme le rappelle encore DESCATOIRE, Ch. «Figures iconographiques: images de la Croix, correspondances typologiques, allégories et vertus ", in Une Renaissance. L'art entre Flandre et Champagne 1150-1250, Paris, 2013 (Catalogue d'exposition) : 73.

18. La pensée typologique établit des correspondances entre des épisodes du Nouveau et de l'Ancien Testament - les seconds étant considérés comme des préfigures, des «types » des premiers.

19. Voir HENRIET, P., « Relire l'autel de Stavelot», in Orfêvrerie septentrionale. XII et XIII ${ }^{e}$ s. L'œuvre de la Meuse II, George, Ph., éd., Liège, Trésor de la Cathédrale (Feuillets de la Cathédrale de Liège), 2016, p. 179-208: 186-187.

\section{RÉSUMÉS}

On se propose de montrer comment l'illustrateur du Bruxellensis 10066-77 est arrivé à bâtir un étonnant rapport de proximité entre texte et images grâce à des choix dont on ne connaît aucun 
autre exemple dans les Physiologi latins et grecs, ni même dans les Bestiaires au sens large. C'est ainsi que l'illustration, accompagnée de nombreuses légendes identificatrices et explicatives, se présente presque comme un double du texte dans la mesure où elle reprend l'essentiel des éléments narratifs, met généralement en image les métaphores et les comparaisons signifiantes et comporte des personnages dont la gestuelle expressive constitue un véritable langage. Elle acquiert ainsi un statut particulier qui l'affranchit en quelque sorte du texte tout en l'enrichissant. Il n'en reste pas moins que la transposition graphique de ce dernier n'a rien de mécanique, et que chacun des deux moyens d'expression conserve sa spécificité tout en concourant, chacun à leur manière, à la fixation de la leçon de théologie ou de morale.

The present paper shows how the illustrator of MS. Bruxelles, Bibliothèque Royale, KBR 10066-77 has built a striking relationship between text and image thanks to choices of which no other example is known in other Greek and Latin copies of the Physiologus, or even in bestiaries in the broad sense. This is why illustration, together with numerous identifying and explanatory captions, is almost a duplicate of the text insofar as it takes up the essence of the narrative elements, generally transposes into images significant metaphors and meaningful comparisons, and includes some characters whose expressive gestures constitute a real language in itself. In this way, the illustration acquires a special status that frees it from the text while at the same time enriching it. Beyond this, the transposition of the text is not mechanical and each of the two means of expression, text and picture, keeps its individual and concurrent identity, each in its own way, for the development of the moral or theological lesson.

\section{INDEX}

Mots-clés : Physiologus, Bestiaires médiévaux, Rapport texte-images, Ms. Bruxelles, Bibliothèque Royale, KBR 10066-77

Keywords : Physiologus, Medieval Bestiaries, Text-image relationship, Ms. Bruxelles, Bibliothèque Royale, KBR 10066-77

\section{AUTEUR}

\section{JACQUELINE LECLERCQ-MARX}

Jacqueline Leclercq-Marx est professeur honoraire d'Histoire de l'art du Moyen Âge occidental, à l'Université Libre de Bruxelles (ULB). La réception de la culture antique dans le monde médiéval jusqu'à 1200 environ - est au cœur de ses recherches qui sont systématiquement fondées sur une double approche iconographique et textuelle. Spécialiste du monstrueux anthropomorphe issu de la mythologie gréco-romaine, elle tente d'en explorer tous les aspects. Parallèlement, elle travaille sur le Physiologus et les Bestiaires. 